\title{
A Novel Treatment for a Han Patient with High Risk of Coronary Obstruction Underwent TAVI Procedure using a Novel Second-Generation Device
}

\author{
Duraid Hamied Naji1,a, Sheng Wang ${ }^{1}$, Zhaoyun Cheng,b*, Zhenwei Ge' ${ }^{1}$, Xianjie Chen1, Yingqiang Guo ${ }^{2}$, Da Zhu² \\ ${ }^{1}$ Department of Cardiovascular Surgery, Henan Provincial People's Hospital, FuwaiCentral China cardiovascular hospital, People's Hospital of \\ Zhengzhou University, Zhengzhou, Henan, 450003, China \\ ${ }^{2}$ Department of Cardiovascular Surgery, West China Hospital of Sichuan University, Chengdu,China. \\ ${ }^{b}$ Postdoctoral in Henan Provincial People's Hospital Heart Centre. Workstation in Fuwai Central China Cardiovascular Hospital, Department \\ of Cardiac Surgery.1, Fu Wai Road, Zhengzhou, Henan 451464, P.R.China C. Key laboratory of Algal Biology, Institute of Hydrobiology, Chinese \\ Academy of Sciences, Wuhan 430072, China \\ ${ }^{a}$ Director and Consultant Surgeon, Department of Cardiac Surgery. Fuwai Central China Cardiovascular Hospital, Henan Provincial People's \\ Hospital Heart Centre.1, Fu Wai Road, Zhengzhou, Henan 451464, P.R.China
}

*Corresponding Author: Zhaoyun Cheng, Department of Cardiovascular Surgery, Henan Provincial People's Hospital, Fuwai Central China cardiovascular hospital, People's Hospital of Zhengzhou University, Zhengzhou, Henan, China.

E-mail: beijingws3@163.com

Received: June 12, 2019; Published: July 04, 2019

DOI: 10.31080/ASMS.2019.03.0341

\section{Abstract}

In this study the obstruction of coronary ostium (CO) is unusual but lethal complication under TAVI procedure it is not normally accepted that a range distance between coronary ostium (CO) and the annulus lower than $10 \mathrm{~mm}$ or in case of sinus of Valsalva diameter around $30 \mathrm{~mm}$, while the existing of bulky counted as a leaflet, this is also in case of the valve in valve implantation, in this situation consider as a risks for coronary occlusion (CO) as for TAVI methodology [1,2]. In TAVAI device using J-Valve ${ }^{\mathrm{TM}}$ system is a brand new second-generation and TAVI device divided into three U-shapes anatomically oriented devices such as "claspers" which could favor conjectural and "self-positioning" valve implantation [3]. Furthermore, Due to the ability to the enclosure and snaffle the main major of leaflets in the main time of valve implantation and in a specific designed extra bear area located on the valve stent. The novelty of this valve is particularly appropriate for the patient who is more in the risk for coronary ostium (CO) such as valve-in-valve implantation. In this study convey the patient of utilizing J-Valve in therapy the case with a high risk of coronary ostium (CO) during TAVI procedure.

Keywords: TAVI; Coronary Ostium (CO); Valve Implantation (VI); J-Valve ${ }^{\mathrm{TM}}$

\section{Case Presentation}

All study subjects were from a female with young age was being referred to our main campus hospital "Fuwai Central China of Cardiovascular Hospital", due to severe aortic stenosis and recurrent episodes of decompensated left heart failure. The patient was with the history of stroke before seven months of the surgery with non-obvious sequelae.

A several in Transthoracic echocardiogram (TTE) were discovered an acute aortic stenosis(AAS) with average pressure gradient of $58 \mathrm{mmHg}$ and aortic valve (AV)slot area around $0.6 \mathrm{~cm}^{2}$ and it significant with Extra-cardiac arterial (ECA) disease involving carotid, intra-cranial and lower limb arteries (LLA) was notified through medical tests such as vascular ultrasound (VU) and MRI angiogram. CT angiogram detected a more calcified aortic valve with aortic annulus diameter $25 \mathrm{~mm}$ comes from annulus perimeter. In addition, CT also appears a low coronary height (Left coronary with height $9.9 \mathrm{~mm}$, right coronary with height $11.1 \mathrm{~mm}$ ), in case of the small aortic sinus with $30 \mathrm{~mm}$ as well as extended aortic valve leaflet with good calcification. In according with interdisciplinary assessment Patient was specific details schedule for transapical aortic valve implantation because of a significantly upon the risk for conventional surgery. TAVI procedure using J-ValveTM prosthesis was well performed via a trans-apical approach. TAVI methodology was well explained and acquired previously [3]. 
The apical puncture diagnosis was obedient with a super-stiff guide-wire set into the slope aorta. As well as the $14 \mathrm{~F}$ sheath was neatly integrated into the left ventricle followed by a balloon catheter. Pre-procedural balloon-valvuloplasty of the native valve was also outright under quick ventricular pacing. In parallel, J-ValveTM with $25 \mathrm{~mm}$ prosthesis was creased into the 27-F Ausper-AS well surrender system, every delivery system was sharply inserted into the left ventricle and very well advanced under using fluoroscopic guidance into a supra-annular position. Three 'U-shaped' graspers were then totally released first and very patient placed into the right identical aortic sinus by gently pulling back the delivery system accolade main leaflets. This Case has The angiogram and then well obtain to assure that all the graspers were designed correctly into every aortic sinus. The valve was recover nicely and back into the annular plan with the response of the graspers and also work without any rapid ventricular pacing. Through the Angiogram we confirmed the optimal valve position with trivial PVL and excellence coronary artery (CA). After all, The suffering patient was moved to the acute care unit. After surgery The patient was in stable and also well discharge, For the first time, CTA and TTE revealed an excellent valve position and function without PVL been improved, coronary artery was successfully protected from a native aortic leaflet by the clipping effect of the clasper.

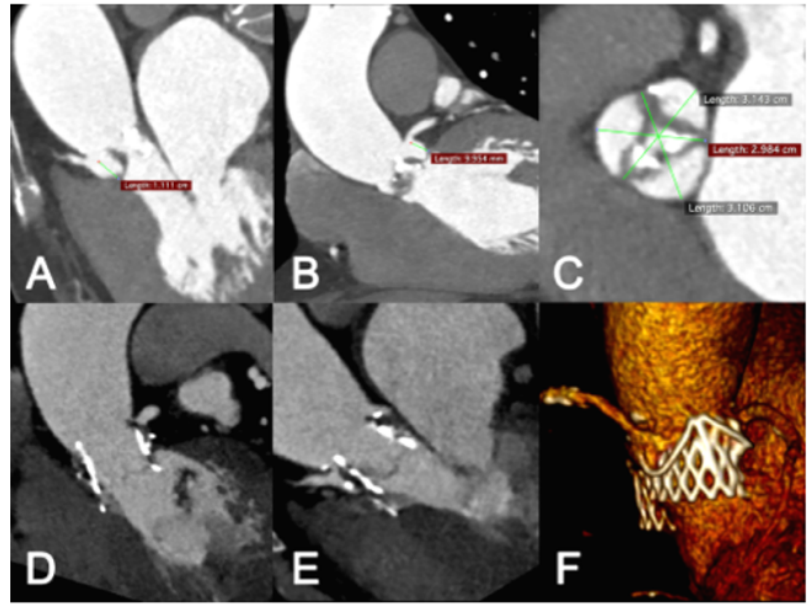

Figure 1: J-Valve implantation in a patient with high risk of coronary obstruction. Upper Panel: Note both left coronary height was all less than $10 \mathrm{~mm}$ with narrow aortic sinus (A-C).Lower Panel: CTA image after valve implantation. Valve was in good position with a patent coronary artery; the clasper of the J-Valve embraced and secured the native aortic valve leaflet then subsequently leaving the coronary ostia freely accessible from aortic leaflet (D-F).
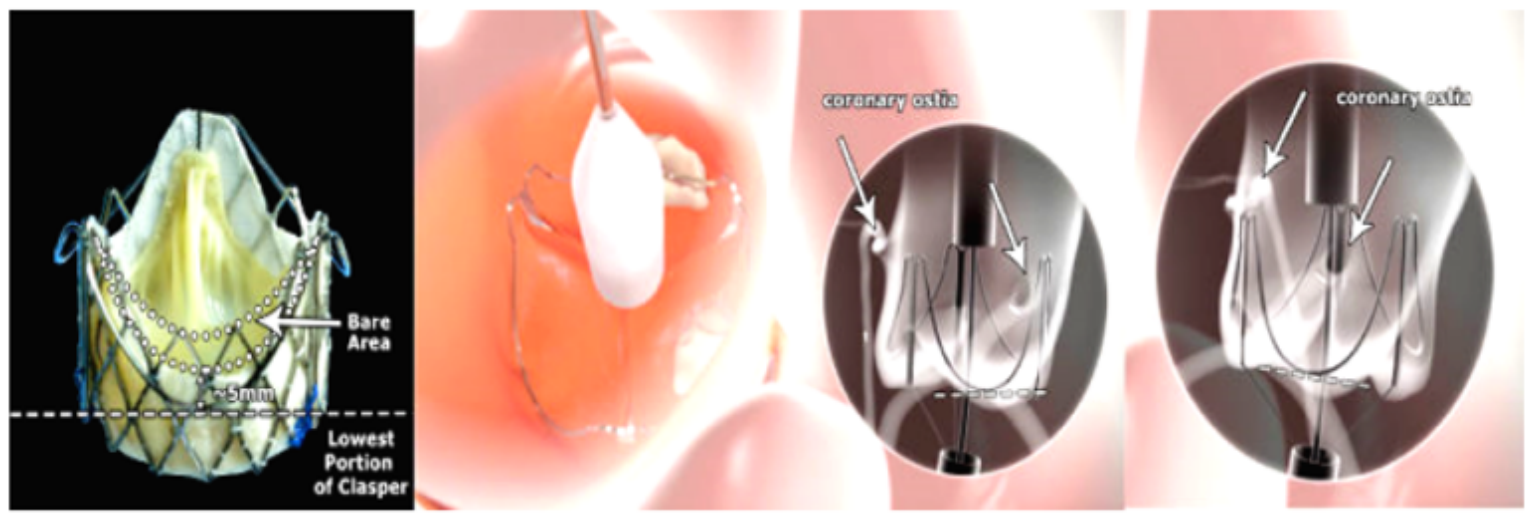

Figure 2: The J-ValveTM system is a new second-generation TAVI device that is featured by three U-shape anatomical orientated devices - "Clasper" encircling around the valve frame. It has a low-profile design with the extra bare mental area in coronary orifice region to minimize the risk of coronary obstruction.

\section{Discussion}

In this large-scale study, we estimate the prospect incidence of coronary obstruction obtained in senior TAVI series and record are comparatively less [1]. We identified in this study that a distance between the coronary ostium and the annulus less than $10 \mathrm{~mm}$ or sinus of Valsalva diameter below $30 \mathrm{~mm}$, existing of bulky leaflet calcification, elongated leaflets as well as aortic valve-in-valve implantation are significant causing high risks for coronary occlusion as for classic TAVI devices such as Edward SAPIEN XT and Medtronic Core Valve system due to their design [2]. All Study subjects were 
expression uncorrelated between the western population and Asian population in this case, Han patient was always associated with low body weight, relatively small aortic root, heavily calcified leaflet as well as low coronary orifice height indeed they need a specific TAVA device.

In summary, To the best of our knowledge, this is the first time that a J-Valve used and it is a new generation of self-expanding lowprofile trans catheter porcine valve that is designed to be delivered transapically. The porcine valve is mounted annularly on the valve stent. Valve has been notable by three main looks like a U-shape in anatomical orientated devices which surrounds around the valve frame. The device has been designed to simplify chromatically and self-positioning valve implantation via detecting the main aortic valve leaflet (AVL). The main design of $U$-shape claspers could folds once and protect the aortic valve leaflet and then automatically left the coronary ostia freely accessible from aortic leaflet; This individual has been notified with Excellent success through using of Jena Valve and Medtronic Engager valve.

\section{Conclusion}

In conclusion, we have detected, The new J-Valve TM prosthesis is a brand new second generation TAVI device in our surgeries. Our study, for the first time in China, this system may provide a safe treatment option for the patient with a high-risk factor of coronary obstruction underwent TAVI procedure in Han population.

\section{Acknowledgments}

We thank the study subjects for their participation and support of this surgery and study reports and all members of the Department of Cardiovascular Surgery team for help and assistance.

\section{Author Contributions}

S.W., Z.Y.C., D. H. N., Z.G., X.C., Y.Q.G., and D.Z., contributed equally to this study. Study concept and design: D.H.N., and Z.Y.C., Acquisition of data: S.W., Z.Y.C., D. H. N., Z.G., X.C., Y.Q.G., and D.Z., Analysis and interpretation of data: D.H.N. Drafting of the manuscript: D.H.N., S.W., Z.Y.C. Critical revision of the manuscript for important intellectual content: D.H.N., Z.Y.C. Statistical analysis: D.H.N. Obtained funding: S.W., Z.Y.C. Study supervision: Z.Y.C., Z.G., X.C., Y.Q.G., and D.Z.

\section{Bibliography}

1. Ribeiro HB., et al. "Predictive factors, management, and clinical outcomes of coronary obstruction following transcatheter aortic valve implantation: insights from a large multicenter registry". JACC: Cardiovascular Interventions 6 (2013): 452461.

2. Ribeiro HB., et al. "Coronary obstruction following transcatheter aortic valve implantation: a systematic review". JACC: Cardiovascular Interventions 6.5 (2013): 452-461.

3. Zhu D., et al. "Treatment of Pure Aortic Regurgitation Using a Second-Generation Transcatheter Aortic Valve Implantation System". Journal of the American College of Cardiology 67 (2016): 2803-2805.

\section{Volume 3 Issue 8 August 2019}

(C) All rights are reserved by Duraid Hamied Naji., et al. 